\title{
Why a D2 gastrectomy plus adjuvant chemotherapy is insufficient in locally advanced gastric cancer
}

\section{Sebastián Solé Z, Francisco E Larsen and Claudio V Solé}

IRAM Clinic, Universidad Diego Portales, Americo Vespucio Norte 1314, Chile 7630370

Correspondence to: Sebastián Z Solé. E-mail: sebasole@gmail.com

\section{Abstract}

This review discusses all the important published evidence regarding adjuvant treatments in locally advanced gastric cancer. In this process it revealed facts that demonstrate the superiority of radiotherapy and concomitant chemotherapy to chemotherapy alone. Some outstanding work that has not yet been published is also discussed.

Keywords: gastric, cancer, stomach, adjuvant, radiotherapy, chemotherapy, gastrectomy

Published: 21/12/2016

Received: 26/07/2016

ecancer 2016, 10:706 DOI: 10.3332/ecancer.2016.706

Copyright: (c) the authors; licensee ecancermedicalscience. This is an Open Access article distributed under the terms of the Creative Commons Attribution License (http://creativecommons.org/licenses/by/3.0), which permits unrestricted use, distribution, and reproduction in any medium, provided the original work is properly cited. 


\section{Introduction}

Surgery was for years the exclusive treatment for gastric cancer, but its results are poor. In the best series, the survival rate is around $30-40 \%$ when there are advanced and compromising locoregional factors. In 1995, the NEJM Fuchs published the results of gastrectomy in patients with compromised serosa and/or lymph nodes. These were not very encouraging as only a five-year survival period was achieved for only $29 \%$ and locoregional failures [1] was observed in $40 \%$. On the other hand, García and collaborators reviewed 423 cases treated with surgery in a hospital in Santiago [2]. The overall survival (OS) rate of the group at five years was 33\%. Patients with stage III gastric cancer had a survival rate of $19.6 \%$ to $38 \%$, which compares favourably with the Memorial Sloan Kettering series published in 2000 [3]. In addition, a group from the University of Valparaíso published the results from 85 patients, who were operated on with R0 resection, resulting in a fiveyear survival rate of $20.7-33.3 \%$ in patients with nodal involvement [4]. Subsequently in the Hospital de la Universidad de Chile, Csendes and colleagues were able to carry out a prospective randomised study of D2 gastrectomy with splenectomy versus the same surgery without a splenectomy. There were no significant differences between the approaches, so they recommend not performing routine splenectomy. When looking at the survival rates of these patients who had very good quality surgery, we can see that the results in stages III range from 20-40\% [5].

\section{Objectives}

This review of scientific literature aims to expose data suggesting that the best adjuvant therapy for patients with advanced non-metastatic gastric cancer is a combination of radiotherapy and concomitant chemotherapy.

\section{Description and analysis of the literature}

The poor results obtained in the series of patients treated exclusively with surgery forced the issue of exploring adjuvant treatments. A collaborative group comprising the IRAM Clinic in Santiago, the Clínica Reñaca, and the Naval Hospital in Viña del Mar led by Dr. Baeza published a study with 52 patients with gastric cancer. They underwent complete resection (R0) and received adjuvant treatment with radiotherapy and concomitant chemotherapy (RTQT). All had nodal or serous involvement. The five-year survival rate was quite encouraging, reaching $54 \%$ in a group of patients in which $86.5 \%$ had nodal involvement. The protocol was not harmless, but it is noteworthy that $100 \%$ of the patients completed it and $73 \%$ of them did so within the established time frame [6]. In 2008, Garrido and colleagues reviewed all the patients treated at the Catholic University with D2 gastrectomy plus RTQT. They compared their results with a historical cohort from their hospital with the same surgery, but without adjuvant treatment. Patients who received RTQT had a better survival rate and no patient discontinued the treatment. They considered that the toxicity of the adjuvant regime was low [7].

The first randomised trial to demonstrate a global survival benefit for an adjuvant treatment in gastric cancer was that of MacDonald published in 2001 [8]. The RTQT decreased locoregional recurrences and this was manifested in a $9 \%$ increase in OS at three years (from 41-50\%). After more than ten years of follow-up, this study updated its data, and the benefit in OS with the highest follow-up persisted without the appearance of late aggregate toxicity. The criticism of this work is that the majority of these patients had resections considered insufficient by the standards of other regions of the world. Therefore, it is questioned if a more radical surgery (D2) could make the use of RT unnecessary. Although D2 resection is recognised as a standard practice in patients with gastric cancer, this intervention has failed to demonstrate a benefit in OS versus less radical dissection (D1). This has been studied in several randomised studies. When grouped in a meta-analysis, it can be seen that the radical intervention is not harmless as it produces a significant increase in hospital stay, postoperative complications, mortality, anastomotic leak, and re-interventions [9].

On the other hand, adjuvant therapy with chemotherapy alone has been evaluated i.e. taking the radiotherapy away from the treatment. The MAGIC study evaluates the use of chemotherapy for six cycles, three preoperative, and three postoperative versus only surgery. It was observed that adding chemotherapy gives a benefit in OS to patients. This despite the fact that the complete protocol was received only 
by $42 \%$ of the experimental arm. It appears that after three cycles of chemotherapy and a gastrectomy, it is difficult to receive more chemotherapy [10]. Another study assessing adjuvance with chemotherapy is the CLASSIC study which is a collaboration between centres in South Korea, China, and Taiwan and published its first results in 2012. In this study, more than a thousand patients were randomly assigned a D2 gastrectomy versus the same surgery and six months of adjuvant chemotherapy with capecitabine and oxaliplatin. There was an OS benefit with adjuvant treatment but only $66.5 \%$ of the patients were able to complete it, and a grade 3-4 toxicity was obtained in $56 \%$ of them. Once again it is verified that adding treatments to the surgery improves its results, but in this case at a high price because of toxicity [11]. The next question is how this treatment is compared with adjuvant RTQT.

The only randomised study comparing chemotherapy versus RTQT as an adjuvant treatment in patients with gastric cancer, i.e. who have had a gastrectomy and D2 dissection, is the ARTIST study. In order to gain all we can learn from this work, we must analyse the three publications of the latter $[12,13,14]$. The main objective of the study was to show a difference in disease-free survival (DFS) (not OS). It was not a large study as it only randomised 458 patients. The RTQT arm was better tolerated and could be completed in $81.7 \%$ of patients versus $75.4 \%$ of patients receiving adjuvant chemotherapy. The three-year DFS was higher for RTQT versus chemotherapy (78\% versus $74 \%)$, but this did not achieve statistical significance even though the $p$-value is close $(p=0.0862)$. The main reason for not achieving a significant $p$-value was that the study included many patients without lymph node involvement, in whom adequate adjuvant chemotherapy is enough. In fact, when analysing the DFS of all patients with node involvement, this was better for those who received RTQT with a significant $p$-value $(p=0.0365)$. Added to this, the adjuvant chemotherapy arm had $3 \%$ more N0 patients, and hence had a better prognosis. The third publication of this study is the most interesting, as it makes a more in-depth analysis of the results. In patients with nodal involvement, locoregional recurrence was $14.5 \%$ for the adjuvant chemotherapy arm versus only $6.4 \%$ in the RTQT arm $(p=0.009)$. We should note that this is in the regions with the best or most radical surgery in the world. Another point to emphasise is the protocol of the radiotherapy that was used. The technique used was anterior-posterior fields. The one used here was a 2D radiotherapy which was extremely old, only slightly homogeneous, inaccurate, and poorly shaped for treatment of targets which were very irregularly shaped (splenic hilum, hepatic hilum, anastomosis, celiac trunk, etc.) and with many healthy organs to avoid irradiating (small intestine, large intestine, kidneys, liver, etc.). They did not use simulation with computed tomography or 3D conformational dosimetry, both standard in radiotherapy throughout Chile for at least a decade. It is noteworthy that even with poor radiation therapy, there has been a benefit in some patients. There is a study from the Princess Margaret Cancer Centre in Toronto which shows that their specialists prefered more tailored treatments such as intensity modulated radiotherapy (IMRT) for this kind of pathology. This is because with this technique the coverage of the region to be irradiated is far better. The region receives the dosage it is meant to and not less, and the doses given to the healthy organs are smaller [15]. It should be noted that this technique is also available in most radiotherapy centres in Chile.

In addition, the ARTIST study highlights the Forest Plot that brings forth the results in the different subgroups. All subgroups show the benefits of RTQT, except for patients without lymph node involvement (see Figure 1).

At the American Society of Clinical Oncology (ASCO) 2016 annual meeting, the initial results of the CRITICS study, which has not yet been published, were presented. This randomised study compares neoadjuvant chemotherapy followed by surgery and subsequently more chemotherapy versus the same neoadjuvant treatment but RTQT after surgery. There were no differences between the arms but only $52 \%$ of the patients [16] randomised to RTQT received it. Whereas in the MAGIC study, after neoadjuvant treatment and surgery, a large number of patients did not tolerate further treatment. We are awaiting the publication of this study.

\section{Future publications}

There are two very interesting studies which are recruiting patients. One is the ARTIST-II (NCT01761461) study which randomises patients with D2 resection and nodal involvement into three-arms, two for chemotherapy and one for RTQT. By 2014, there were already 118 randomised patients. Another very interesting study is that of TOPGEAR (NCT01924819), which for the first time evaluates neoadjuvant RTQT treatment in gastric cancer versus neoadjuvant chemotherapy alone. By 2015, there were 170 randomised patients. 


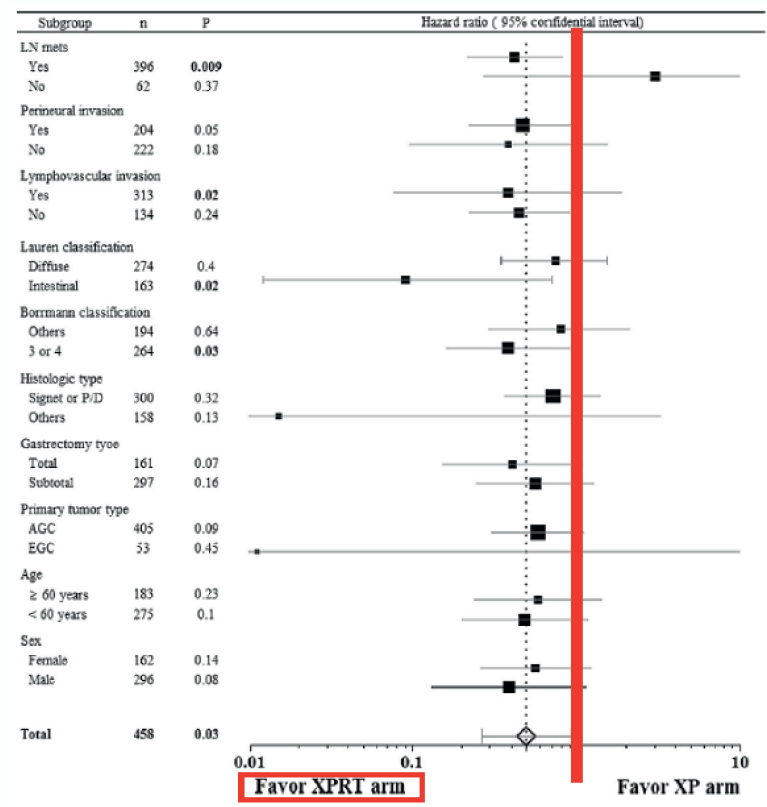

Figure 1. ARTIST trial subgroup analysis for disease-free survival. To the left of the red line, results favor RTQT. To the right of the red line, results favor chemotherapy.

\section{Conclusion}

D2 gastrectomy plus adjuvant chemotherapy is not sufficient for locally advanced gastric cancer. The only comparison of this strategy versus adjuvant RTQT (ARTIST) shows that with surgery and chemotherapy, locoregional recurrence is $14.5 \%$ versus only $6.4 \%$ when RTQT is carried out $(p=0.009)$. In addition, the RTQT scheme has less toxicity and can be completed by a greater proportion of patients. We must also consider that present radiotherapy worldwide has improved greatly with respect to radiotherapy used at the time of the randomised trials which even then had proved to be beneficial for this pathology.

\section{References}

1. Fuchs CS and Mayer RJ (1995) Gastric carcinoma N Engl J Med 333(1) 32-41 DOI: 10.1056/NEJM199507063330107 PMID: $\underline{7776992}$

2. García C et al (2007) Results of the surgical treatment of gastric cancer. Analysis of 423 cases Rev Méd Chile 135(6) 687-95

3. Martin RC 2nd et al (2002) Extended local resection for advanced gastric cancer: increased survival versus increased morbidity Ann Surg 236(2) 159-65 DOI: 10.1097/00000658-200208000-00003 PMID: 12170020 PMCID: 1422561

4. Cenitagoya GF, Bergh CK and Klinger-Roitman J (1998) A Prospective Study of Gastric Cancer. 'Real' 5-year survival rates and mortality rates in a country with high incidence Dig Surg 15(4) 317-22 DOI: 10.1159/000018645 PMID: 9845606

5. Csendes A et al (2002) A prospective randomized study comparing D2 total gastrectomy versus D2 total gastrectomy plus splenectomy in 187 patients with gastric carcinoma Surgery 131(4) 401-7 DOI: 10.1067/msy.2002.121891 PMID: 11935130 
6. Baeza MR et al (2001) Adjuvant radiochemotherapy in the treatment of completely resected, locally advanced gastric cancer Int J Radiat Oncol Biol Phys 50(3) 645-50 DOI: 10.1016/S0360-3016(01)01467-5 PMID: 11395231

7. Garrido M et al (2008) Radio-postoperative chemotherapy in locally advanced gastric cancer Rev Méd Chile 136(7) 844-850

8. MacDonald JS et al (2001) Chemoradiotherapy after surgery compared with surgery alone for adenocarcinoma of the stomach or gastroesophageal junction N Enlg J Med 345(10) 725-30 DOI: 10.1056/NEJMoa010187

9. Memon MA et al (2011) Meta-analysis of D1 versus D2 gastrectomy for gastric adenocarcinoma Ann Surg 253(5) 900-11 DOI: 10.1097/SLA.0b013e318212bff6 PMID: 21394009

10. Cunningham $\mathrm{D}$ et al (2006) Perioperative chemotherapy versus surgery alone for resectable gastroesophageal cancer $N$ Engl J Med 355(1) 11-20 DOI: 10.1056/NEJMoa055531 PMID: 16822992

11. Noh SH et al (2014) Adjuvant capecitabine plus oxaliplatin for gastric cancer after D2 gastrectomy (CLASSIC): 5-year follow-up of an open-label, randomized phase 3 trial Lancet Oncol 15(12) 1389-96 DOI: 10.1016/S1470-2045(14)70473-5 PMID: $\underline{25439693}$

12. Lee J et al (2012) Phase III trial comparing capecitabine plus cisplatin versus capecitabine plus cisplatin with concurrent capecitabine radiotherapy in completely resected gastric cancer with D2 lymph node dissection: the ARTIST trial $J$ Clin Oncol 30(3) 268-73 DOI: $\underline{10.1200 / J C O .2011 .39 .1953}$

13. Park SH et al (2015) Phase III trial to compare adjuvant chemotherapy with capecitabine and cisplatin versus concurrent chemoradiotherapy in gastric cancer: final report of the adjuvant chemoradiotherapy in stomach tumors trial, including survival and subset analyses $J$ Clin Oncol 33(28) 3130-6 DOI: 10.1200/JCO.2014.58.3930 PMID: 25559811

14. Yu Jl et al (2015) Effects of adjuvant radiotherapy on completely resected gastric cancer: A radiation oncologist's view of the ARTIST randomized phase III trial Radiother Oncol 117(1) 171-7 DOI: 10.1016/j.radonc.2015.08.009 PMID: 26299196

15. Ringash J et al (2005) IMRT for adjuvant radiation in gastric cancer: a preferred plan? Int J Radiat Oncol Biol Phys 63(3) 732-8 DOI: $10.1016 / j . j$.jrobp.2005.03.013 PMID: 15978742

16. Verheij $\mathrm{M}$ et al (2016) A multicenter randomized phase III trial of neo-adjuvant chemotherapy followed by surgery and chemotherapy or by surgery and chemoradiotherapy in resectable gastric cancer: First results from the CRITICS study $\mathrm{J}$ Clin Oncol 34 (suppl; abstr 4000) 